\title{
Integrating Polarized Light Microscopy and Spectroscopy for Improved Identification of Suspected White Powder Bioterrorism
}

\author{
G.L. Shearer
}

McCrone Associates, 850 Pasquinelli Drive, Westmont, IL 60559

The general public envisions suspected weapons of mass destruction (WMD) as white powders, which must be contained and investigated by first responders. These white powder investigations require rapid, specific identifications that are normally carried out on site by a First Responder Team. To date, most white powder incidents have not been hazardous, but inevitably, the question posed to investigators is "If it is not hazardous, what is it?" Thus, a complete and accurate identification of the white powder is needed. The optimal analytical approach when faced with an unknown sample is to start with optical examination to characterize the sample. Then, the most appropriate analytical techniques may be selected based on the optical properties of the unknown. However, first responders have to handle suspect samples safely, and may not be able to start with an optical examination. In many cases, Fourier transform infrared spectroscopy (FTIR) is performed initially, along with other biochemical tests, and light microscopy is performed afterwards. The purpose of this paper is to demonstrate how polarized light microscopy (PLM) can be used in conjunction with vibrational spectroscopy techniques, primarily FTIR, to rapidly and more completely identify unknown white powders.

Light microscopy can be used to provide better specificity for materials that have similar infrared spectra. One such example is amorphous silicates. In Fig. 1, the FTIR spectra of silica gel, glass, pumice and Aerosil $200^{\circledR}$ (fumed silica) are compared. The spectra of these amorphous silicate materials are very similar. However, the same four amorphous silicates have different optical properties. Fig. 1 shows plane polarized light images of the same four compounds [1]. It is immediately clear that the fumed silica or Aerosil is very different. The material is isotropic and has an extremely small particle size (about $12 \mathrm{~nm}$ ). What can be seen at $400 \mathrm{X}$ are agglomerates of 2 to 10 micrometers. Isotropicity and fine particle size are indicators of fumed silica. It is important to be able to distinguish fumed silica from other silica as it is a possible aerosol carrier of anthrax.

Mixtures present a particular challenge for FTIR, because they can produce very complex spectra that are not always uniform and may be difficult to identify using library searching. This can be illustrated with baking powder, which is a mixture of corn starch, sodium bicarbonate, sodium aluminum sulfate (soda alum) and calcium acid phosphate. Figure 2 presents two FTIR spectra that were prepared by grinding the sample to make a uniform sample. The top spectrum is similar to corn starch and the bottom spectrum is fairly consistent with sodium bicarbonate or baking soda, but in each case, neither is a perfect match. PLM analysis can readily differentiate the components in baking powder. The PLM image in Fig. 2 clearly demonstrates three distinct particle types.

Further examples that illustrate how combining PLM with vibrational spectroscopy (FTIR and Raman) enables more complete identification will be presented.

[1] The author gratefully acknowledges the McCrone Atlas of Microscopic Particles (www.mccroneatlas.com) for permission to reproduce the photomicrographs. 

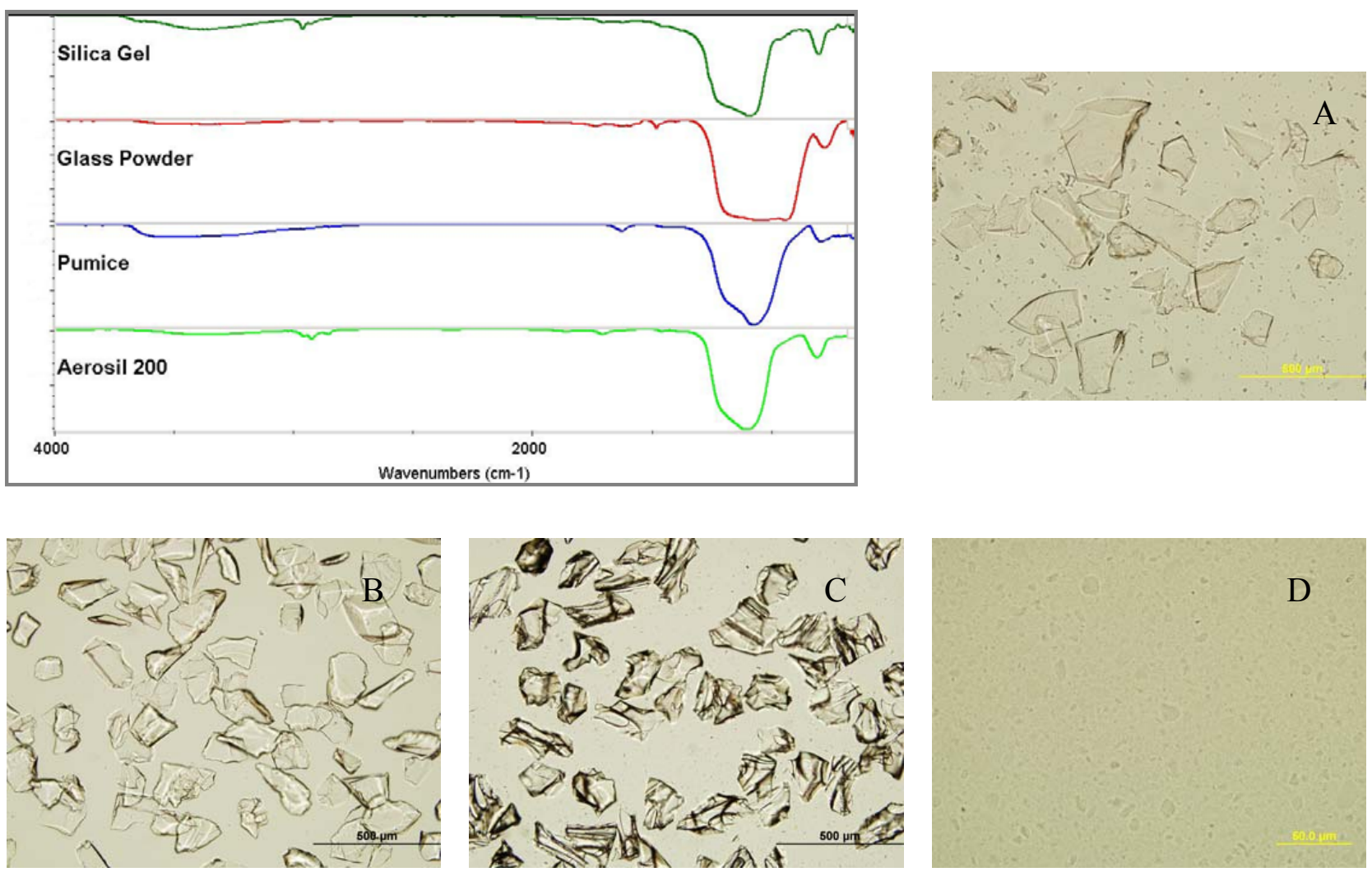

FIG. 1. (top left) The FTIR spectra of silica gel, glass, pumice and Aerosil (fumed silica) are compared. The PLM images of (A) silica gel, (B) glass, (C) pumice and (D) Aerosil (fumed silica). The particles observed in image $\mathrm{D}$ are actually agglomerates of very fine particles.
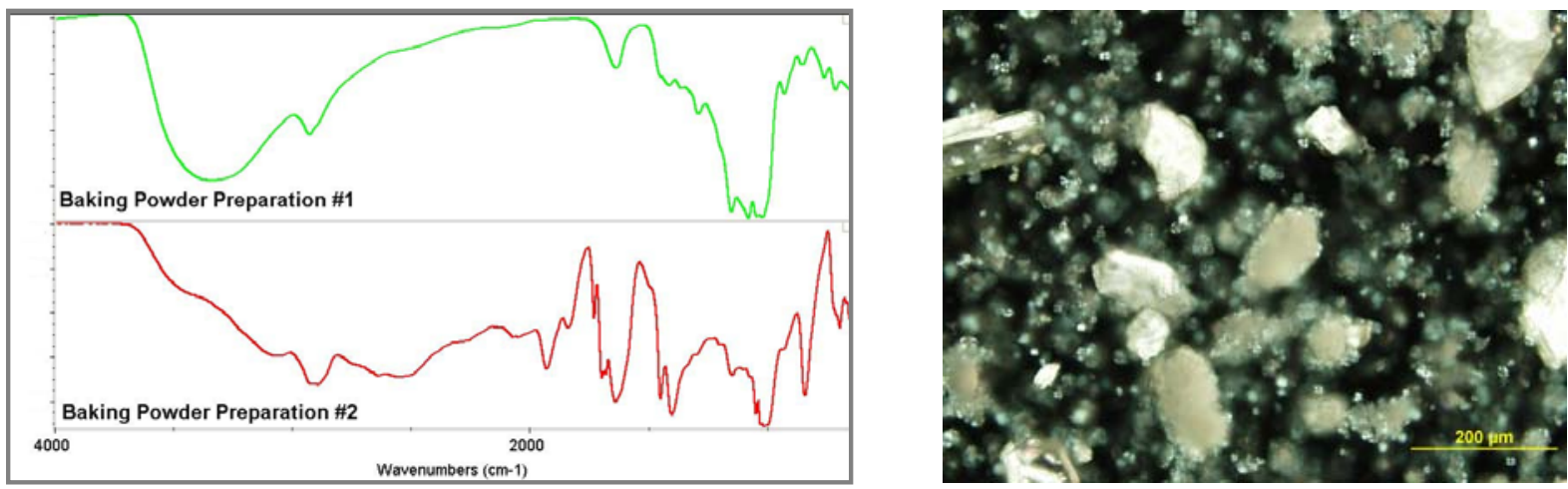

FIG. 2. (Left) FTIR spectra of two different baking powder preparations (infrared microscope transmission mode). The top spectrum is indicative of starch. The bottom spectrum is more similar to sodium bicarbonate. The PLM image (right) clearly shows three particle types: the larger birefringent sodium bicarbonate particles, the white, isotropic ball-shaped sodium aluminum sulfate (soda alum) particles and the corn starch particles. 